\title{
Fatores associados à satisfação com a vida em idosos cuidadores e não cuidadores
}

\author{
Factors associated with satisfaction with life \\ among elderly caregivers and non-caregivers
}

\author{
Monica Regina Scandiuzzi Valente Tomomitsu ${ }^{1}$ \\ Monica Rodrigues Perracini ${ }^{2}$ \\ Anita Liberalesso Neri ${ }^{3}$
}

${ }^{1}$ Programa de Pós-

Graduação em

Gerontologia, Faculdade

de Ciências Médicas,

Universidade Estadual de

Campinas (Unicamp).

Av. Bertrand Russell 801,

Barão Geraldo. 13.083-

970 Campinas SP Brasil.

monicatomomitsu@

gmail.com

${ }^{2}$ Conselho Superior de

Ensino, Pesquisa e Extensão,

Universidade Cidade de São

Paulo.

${ }^{3}$ Departamento de

Psicologia Educacional,

Faculdade de Educação,

Unicamp.
Abstract This article seeks to investigate associations between satisfaction with life and sociodemographic variables, health conditions, functionality, social involvement and social support among elderly caregivers and non-caregivers, as well as between satisfaction and the intensity of stress in the caregiver group. A sample of 338 caregivers was selected according to two items of the Brazilian version of the Elders Life Stress Inventory. A comparison-group of elderly non-caregivers was selected at random, with a similar gender, age and income profile. Data were derived from self-reported questionnaires and scales. Elderly caregivers with low levels of satisfaction and high levels of stress revealed more symptoms of insomnia, fatigue, diseases and worse IADL performance. Those with greater satisfaction and less stress revealed a good level of social support. Insomnia, depression and fatigue were associated with low satisfaction among caregivers, and with fatigue, depression and low social support among non-caregivers. It was considered relevant that instrumental, psychological and informative support can improve the quality of life and the quality of care provided by elderly caregivers, especially if they are affected by unfavorable health and psychosocial conditions and low satisfaction with life.

Key words Caregivers, Stress, Subjective well-being, Health of the elderly
Resumo O objetivo do artigo é investigar associações entre a satisfação com a vida e variáveis sociodemográficas, condições de saúde, funcionalidade, envolvimento social e suporte social em idosos cuidadores e não cuidadores, e entre satisfação $e$ intensidade do estresse no grupo de cuidadores. A amostra foi composta por 338 cuidadores selecionados por dois itens do Elders Life Stress Inventory, versão brasileira. A partir de sorteio aleatório, foi composto um grupo-comparação de idosos não cuidadores, com a mesma distribuição por gênero, idade e renda dos cuidadores. Os dados foram derivados de medidas de autorrelato (questionáriose escalas). Idosos cuidadores com menor satisfação e maior estresse apresentaram mais sintomas de insônia, de fadiga, de doenças e pior desempenho AIVD. Aqueles com maior satisfação e menor estresse apresentaram bom nível de suporte social. Insônia, depressão e fadiga associaram-se com baixa satisfação entre os cuidadores; fadiga, depressão e menor suporte social entre os não cuidadores. Condições de saúde e psicossociais desfavoráveis e baixo nível de satisfação podem limitar a qualidade de vida e do cuidado oferecido por cuidadores familiares idosos, o que sugere a relevância de oferecer-lhes suporte instrumental, psicológico e informativo.

Palavras-chave Cuidadores, Estresse, Bem-estar subjetivo, Saúde do idoso 


\section{Introdução}

A literatura psicológica e sociológica sobre bem estar subjetivo estabelece que níveis mais elevados de satisfação com a vida favorecem boas condições de saúde física e mental e boas relações sociais e são um importante indicador de bem-estar e qualidade de vida em adultos e idosos ${ }^{1}$. Fujita e Diener ${ }^{2}$ relataram que a satisfação com a vida não sofre grandes flutuações ao longo do curso de vida, mas Mroczek e Spiro ${ }^{3}$, Gerstorf et al. ${ }^{4}$ e Hernandez et al. ${ }^{5}$ observaram crescimento dos níveis de satisfação com a vida entre os 40 e os 70 anos de idade, seguido por um período de estabilidade e por tendência ao declínio após os 80 anos $^{6}$. Segundo estes autores, este decréscimo está correlacionado com maior número de sintomas clínicos, baixo senso de controle, presença de sintomas depressivos, estar ou morar sozinho, pior desempenho funcional e idade avançada. O nível de satisfação pode declinar na presença de eventos de vida que desafiam o equilíbrio dos idosos mas, graças aos recursos psicológicos presentes na velhice ${ }^{7,8}$, é comum os idosos se adaptarem e retornarem ao nível basal de satisfação com a vida, depois de algum tempo de exposição a tais eventos ${ }^{1}$.

Prestar cuidado é um evento de vida que pode produzir desfechos negativos tais como depressão, pior condição de saúde física e níveis mais baixos de bem estar ${ }^{9} \mathrm{em}$ adultos e idosos, mas não tem uma trajetória idêntica e nem linear para todos os envolvidos. Muitos cuidadores são capazes de lidar bem com as tarefas do cuidar, sem se sentirem onerados. Borg e Hallberg ${ }^{10}$ observaram nível similar de satisfação com a vida entre cuidadores idosos pouco comprometidos com o cuidado e não cuidadores, mas encontraram diferenças significativas em satisfação com a vida entre cuidadores muito onerados por tarefas de cuidado e não cuidadores. Pinquart e Sörensen ${ }^{9}$ observaram que o grau de dependência física e cognitiva e a presença de distúrbios de comportamento dos idosos que recebem cuidados correlacionam-se com nível baixo de bem-estar subjetivo, principalmente quando o cuidar implica em grande ônus físico. No entanto, Borg e Hallberg ${ }^{10}$ relataram que estes desfechos parecem depender das condições sociais e de saúde física e psicológica, pois verificaram níveis mais elevados de satisfação com a vida entre idosos cuidadores que detinham boas condições de saúde, recursos financeiros suficientes e uma rede de apoio social eficaz. Os mesmos autores observaram que os fatores associados à redução da satisfação com a vida entre idosos cuidadores são má qualidade do sono, saúde percebida como ruim, excesso de tarefas de cuidar e alto grau de dependência física do ente cuidado.

Há evidências robustas sobre o papel protetor do suporte social percebido e de bons níveis de envolvimento social, em relação à satisfação com a vida entre idosos cuidadores ${ }^{11}$, bem como entre idosos não cuidadores ${ }^{1}$. A continuidade das atividades sociais e a disponibilidade de apoio social contribuem para a manutenção do bem estar subjetivo entre cuidadores de idosos com demência, provavelmente porque as habilidades associadas à sociabilidade e o suporte social percebido geram mais emoções positivas, proporcionam melhor ajustamento emocional, melhoram a saúde e diminuem o nível de estresse ${ }^{11,12}$.

Em idosos mais jovens, a dependência em atividades instrumentais de vida diária (AIVD) é um marcador de declínio funcional e cognitivo. Pode aguçar a percepção de estresse a eventos de vida tais como o cuidado, devido à percepção de perda de autonomia. Nesses casos, pode ocorrer declínio na satisfação com a vida, mesmo na presença de apoio social. Entre idosos muito idosos, eventos como o falecimento do cônjuge ou de amigos próximos e o estresse associado à piora dos próprios sintomas clínicos afetam mais a diminuição do nível de satisfação com a vida do que a incapacidade funcional ${ }^{13}$. Gana et al. ${ }^{14}$ sugeriram que o nível de satisfação com a vida não é homogêneo, podendo sofrer alterações ao longo do curso da vida, em face da intensidade atribuída aos eventos estressantes, dos relacionamentos sociais e do senso de controle.

O processo de envelhecimento populacional em curso no Brasil tenderá a acionar, de forma cada vez mais intensa, os recursos da família e da sociedade para cuidar de idosos doentes e dependentes. Devido ao aumento da longevidade, entre os cuidadores tenderá a aumentar o número de idosos atendendo a outros idosos, que são seus cônjuges ou progenitores. Então, será de crescente importância que existam familiares em condições de proporcionar cuidados de boa qualidade a idosos física e cognitivamente dependentes. De acordo com dados de pesquisas internacionais, a satisfação com a vida favorece a saúde física, a cognição, a saúde psicológica e as relações sociais dos cuidadores. Como há poucos dados brasileiros sobre as relações entre essas variáveis em idosos e comparando idosos cuidadores e não cuidadores com relação a elas, é relevante realizar pesquisas a este respeito envolvendo idosos brasileiros. Os objetivos deste estudo foram: comparar idosos que prestam cuidado no contexto familiar com idosos não cuidadores, quanto ao nível de satisfação com a vida, condições de saúde, funcionalidade 
Este estudo envolveu idosos com 65 anos e mais residentes em três cidades brasileiras, Parnaíba (PI), Campinas (SP) e Ivoti (RS), cujos dados compunham o banco eletrônico do Estudo FIBRA (acrônimo de Fragilidade em Idosos Brasileiros), polo Unicamp, e sobre relações entre fragilidade, condições sociodemográficas, psicossociais, de saúde, cognitivas e de funcionalidade. Em cada cidade, a seleção dos idosos foi feita a partir de amostragem aleatória simples dos setores censitários urbanos, em cujos domicílios foram recrutadas cotas de homens e mulheres que neles tinham residência permanente e que satisfaziam os critérios de elegibilidade (ausência de limitações graves de natureza física, sensorial, cognitiva, de mobilidade ou de comunicação e residência permanente no setor censitário e no domicílio). Foram recrutadas cotas de homens e de mulheres de quatro grupos de idade (65-69, 70-74, 75-79 e 80 anos e mais), cotas essas proporcionais às observadas nos mesmos grupos de idade e sexo da população urbana de cada cidade.

$\mathrm{Na}$ fase inicial da coleta de dados, após a coleta de dados sociodemográficos, clínicos, antropométricos, de fragilidade e de rastreio de demências, foram excluídos os idosos que pontuaram abaixo da nota de corte no Mini Exame do Estado Men$\mathrm{tal}^{15}$. Os remanescentes participaram do protocolo completo de coleta de dados. O estudo foi conduzido segundo as normas da última Declaração de Helsinki para pesquisa com seres humanos, e foi aprovado pelo Comitê de Ética em Pesquisa da Faculdade de Ciências Médicas (CEP/FCM). Todos os participantes assinaram um termo de consentimento livre e esclarecido. Detalhes metodológicos e sobre as características sociodemográficas das amostras podem ser encontrados em Neri et al. ${ }^{16}$.

\section{Participantes}

Entre os 1.581 idosos que integravam as amostras das três cidades, 1.139 pontuaram acima da nota de corte no Mini Exame do Estado Mental ${ }^{16}$, satisfazendo o critério de inclusão em medidas que incluíam as de interesse para o presente estudo. Dentre eles, 338 satisfaziam a exigência de terem sido cuidadores familiares de outros idosos, ao longo dos 5 anos anteriores à entre-

vista, evidenciada por resposta afirmativa a dois itens da versão brasileira do The Elders Life Stress Inventory - ELSI ${ }^{17,18}$. Esses itens investigavam se os respondentes haviam cuidado do/a cônjuge ou companheiro/a, de pais ou de sogros idosos nos últimos cinco anos. Dentre os que responderam negativamente, ou seja, não cumpriam essa condição (801), foram sorteados 338 para integrar um grupo-comparação de idosos não cuidadores, composto por subgrupos de tamanho similar aos do grupo de cuidadores, considerando-se as variáveis gênero, idade e nível de renda familiar.

\section{Instrumentos}

As variáveis de interesse para este projeto foram investigadas mediante as condições que se seguem.

1) Satisfação com a vida. Foi avaliada mediante um questionário contendo um item sobre a satisfação global com a vida, um item sobre a satisfação em comparação com os outros da mesma idade seis itens sobre a satisfação referenciada aos domínios memória; capacidade funcional e de solução de problemas, amizades e relações familiares, ambiente, acesso a serviços de saúde e meios de transporte ${ }^{19}$. Eram itens escalares de 3 pontos e a pontuação total podia variar de 1 a 24 . Foi calculada a mediana dos escores da amostra. Aqueles que pontuaram $<21$ foram considerados com menor satisfação com a vida e os que pontuaram $>22$ com maior satisfação com a vida. $\mathrm{O}$ alfa de Cronbach foi de 0.729 .

2) Idade, gênero e renda familiar. Três questões de autorrelato, cujos resultados eram anotados pelos avaliadores no formulário de pesquisa (data de nascimento, gênero masculino $\mathrm{x}$ feminino e renda familiar em valores brutos). As idades foram agrupadas em 4 faixas: 65-69; 70-74; 75-79, e 80 e + . Os valores de renda foram agrupados em 5 faixas de salários mínimos $(\mathrm{SM}):<1 ; 1,1$ a $3 ; 3,1$ a $5 ; 5,1$ a 10 , e > 10 SM.

3) Intensidade do estresse associado ao prestar cuidados. Foi avaliada por meio de item único de natureza escalar de 5 pontos, em que 1 correspondia a menor estresse $)^{17,18}$, aplicada aos idosos cuidadores, a qual perguntava qual tinha sido a intensidade do estresse vivenciado na situação.

4) Número de doenças. Avaliado por meio de 9 itens dicotômicos que investigavam se algum médico havia dito que o participante tinha as seguintes doenças crônicas: doença do coração, hipertensão, AVC/isquemia/derrame; diabetes mellitus, câncer, artrite ou reumatismo, doenças dos pulmões; depressão e osteoporose. As respos- 
tas afirmativas foram contadas e foram criadas três categorias: zero, 1 ou 2 e 3 ou mais doenças.

5) Fadiga. Avaliada por dois itens escalares de autorrelato extraídos da Center for Epidemiological Studies-Depression $(C E S-D)^{20}$. Foram considerados com fadiga os idosos que responderam sempre ou na maioria das vezes a qualquer um ou a ambos os itens.

6) Sintomas depressivos. Avaliados por meio da Escala de Depressão Geriátrica ${ }^{21}$ com 15 itens com nota de corte $\geq 6$. O coeficiente de confiabilidade interna (alfa de Cronbach) para esta escala foi de 0.734 .

7) Insônia. Avaliada por quatro questões de autorrelato, que comportavam respostas sim ou não, extraídas do Perfil de Saúde de Nottingham $(\mathrm{PSN})^{22}$. Foram considerados como portadores sintomas de insônia aqueles que responderam positivamente a qualquer um dos quatro itens.

8) Atividades instrumentais de vida diária (AIVD). Sete itens de autorrelato baseados na escala de Lawton e Brody ${ }^{23}$, que avaliaram o nível de independência para AIVDs. Havia três possibilidades de respostas: totalmente independente; necessitado de alguma ajuda; necessitado de ajuda total, para fazer as coisas do dia-a-dia. As respostas foram agrupadas em duas categorias: independência, quando os idosos responderam não precisar de nenhuma ajuda e dependência, quando relataram precisar de ajuda parcial ou total.

9) Envolvimento em atividades avançadas de vida diária (AAVD). Foram avaliadas mediante um questionário ${ }^{24,25}$ contendo 13 itens de autorrelato: fazer e receber visitas, ir à igreja, ir a festas e a eventos culturais, realizar dança de salão, fazer viagens curtas e longas, dirigir automóvel, desempenhar trabalho remunerado e voluntário, participar de universidade da terceira idade e de centro de convivência, e participar de diretorias ou conselhos de sociedades. Para cada um dos itens havia três alternativas de resposta: nunca fiz, ainda faço e parei de fazer. Foram desconsideradas as atividades que os idosos nunca haviam realizado e contada a frequência das que continuavam a desempenhar. Com base na mediana, foram criadas duas categorias: $\leq 5$ e $\geq 6$ AAVD mantidas.

10) Suporte social percebido. Foram aplicados cinco itens semanticamente adaptados para o português do inventário ISEL - Interpersonal Support Evaluation List ${ }^{26}$, cada um com quatro intensidades. A pontuação varia de 1 a 20 . Com base na mediana foram criados dois grupos: $\leq 18$ para os com baixo suporte social percebido e $\geq 19$ para os com alto suporte social percebido. O coeficiente de confiabilidade interna dos itens foi de 0.756 .

\section{Análise de dados}

Foram adotados testes não paramétricos para as análises comparativas, uma vez que os dados não aderiram à distribuição normal avaliada pelo teste Kolmogorov-Smirnov (qui-quadrado ou exato de Fisher para a variáveis categóricas e Mann-Whitney e Kruskal-Wallis para as ordinais). Para estudar os fatores associados à satisfação com a vida entre os cuidadores e os não cuidadores foram feitas análises de regressão logística univariada e multivariada, estas com critério stepwise de seleção de variáveis. O nível de significância adotado para os testes estatísticos foi de $\mathrm{p}<0.05$.

\section{Resultados}

Os grupos de cuidadores e não cuidadores eram estatisticamente equivalentes quanto a gênero, idade e renda familiar. Em cada grupo, 65,7\% eram mulheres, 39,3\% tinham idade de 65 a 69 anos, $37,6 \%$ de 70 a $74,16,3 \%$ de 75 a 79 e $6,8 \%$ de 80 anos e mais; $7,5 \%$ relataram ter renda familiar $\leqq 1$ SM, $45,7 \%$ entre 1,1 e 3 SM; $19,3 \%$ entre 3,1 e 5 SM, $12,1 \%$ de 5,1 a 10 SM e $10,2 \%$, mais do que $10 \mathrm{SM}$. Nos dois grupos, a idade variou entre 65 a 90 anos e a média foi de $71.50( \pm 4.81)$ anos. A renda familiar média foi igual a 3.84 SM mensais $( \pm 4.30)$.

Estiveram envolvidos em cuidados nos cinco anos anteriores à entrevista 338 idosos, correspondente a $29,67 \%$ do total de idosos entrevistados (1.139). Dentre eles, $76.3 \%$ prestavam cuidados ao cônjuge, $15.1 \%$ aos pais e $7.7 \%$ ao cônjuge e aos pais. A intensidade média do estresse gerado pela experiência do cuidado vivenciado por eles foi 3.84 ( \pm 1.35 ); $64,8 \%$ pontuaram 4 ou 5 na escala de intensidade de estresse gerado pelo cuidado (alto estresse) e 35,2\%, pontuaram de 1 a 3 (baixo estresse).

Foram observados mais idosos cuidadores com nível alto de estresse do que com baixo nível de estresse. Entre os cuidadores com maior estresse havia mais idosos com necessidade de ajuda para o desempenho de AIVD e com queixas de insônia do que no grupo de não cuidadores. Não foram observadas diferenças estatisticamente significativas entre as frequências de idosos dos três grupos nas demais variáveis (Tabela 1 ).

A Tabela 2 oferece dados comparativos sobre o nível de satisfação com a vida (maior x menor) dos cuidadores com maior e com menor estresse associado ao prestar cuidados e dos não cuidadores. Entre os cuidadores com menor satisfação e 
Tabela 1. Idosos cuidadores e não cuidadores conforme a satisfação com a vida, as condições de saúde, a funcionalidade em AIVD, o envolvimento em AAVD e o suporte social percebido. Estudo FIBRA em Campinas, Parnaíba e Ivoti, 2008-2009.

\begin{tabular}{|c|c|c|c|c|}
\hline \multirow[b]{2}{*}{ Variáveis } & \multicolumn{2}{|c|}{ Cuidadores } & \multirow{2}{*}{$\begin{array}{l}\text { Não cuidadores } \\
(\mathbf{n}=338)\end{array}$} & \multirow[b]{2}{*}{$\mathrm{p}$} \\
\hline & $\begin{array}{l}\text { Menor estresse } \\
\quad(n=109)\end{array}$ & $\begin{array}{l}\text { Maior estresse } \\
\quad(n=197)\end{array}$ & & \\
\hline Satisfação com a vida & & & & 0.367 \\
\hline Menor $(<21)$ & $59(54.1 \%)$ & $111(56.4 \%)$ & $166(49.1 \%)$ & \\
\hline Maior $(>22)$ & $49(45.0 \%)$ & $83(42.1 \%)$ & $160(47.3 \%)$ & \\
\hline Não responderam & $1(0.9 \%)$ & $3(1.5 \%)$ & $12(3.6 \%)$ & \\
\hline Número de doenças & & & & 0.526 \\
\hline Zero & $11(10.1 \%)$ & $24(12.2 \%)$ & $36(10.7 \%)$ & \\
\hline 1 ou 2 & $54(49.5 \%)$ & $82(41.6 \%)$ & $166(49.1 \%)$ & \\
\hline 3 ou mais & $44(40.4 \%)$ & $91(46.2 \%)$ & $136(40.2 \%)$ & \\
\hline Fadiga & & & & 0.072 \\
\hline Não & $86(78.9 \%)$ & $139(70.6 \%)$ & $264(78.1 \%)$ & \\
\hline $\operatorname{Sim}$ & $21(19.3 \%)$ & $56(28.4 \%)$ & $69(20.4 \%)$ & \\
\hline Não responderam & $2(1.8 \%)$ & $2(1.0 \%)$ & $5(1.5 \%)$ & \\
\hline Sintomas depressivos & & & & 0.180 \\
\hline Não $(<5)$ & $94(86.2 \%)$ & $152(77.6 \%)$ & $269(79.6 \%)$ & \\
\hline $\operatorname{Sim}(>6)$ & $15(13.8 \%)$ & $44(22.4 \%)$ & $69(20.4 \%)$ & \\
\hline Insônia & & & & 0.021 \\
\hline Não & $50(45.9 \%)$ & $73(37.1 \%)$ & $167(49.4 \%)^{\mathrm{a}}$ & \\
\hline Sim & $59(54.1 \%)$ & $124(62.9 \%)^{\mathrm{a}}$ & $171(50.6 \%)$ & \\
\hline Desempenho de AIVD & & & & 0.015 \\
\hline Dependência parcial & $40(36.7 \%)$ & $93(47.2 \%)^{b}$ & $117(34.6 \%)$ & \\
\hline Independência & $69(63.3 \%)^{b}$ & $104(52.8 \%)$ & $220(65.1 \%)^{b}$ & \\
\hline Não responderam & $0(0.0 \%)$ & $0(0.0 \%)$ & $1(0.3 \%)$ & \\
\hline AAVD interrompidas & & & & 0.140 \\
\hline$<2$ & $18(16.5 \%)$ & $44(22.3 \%)$ & $95(28.1 \%)$ & \\
\hline $2-3$ & $56(51.4 \%)$ & $93(47.2 \%)$ & $143(42.3 \%)$ & \\
\hline$>3$ & $35(32.1 \%)$ & $60(30.5 \%)$ & $100(29.6 \%)$ & \\
\hline Suporte social & & & & 0.699 \\
\hline Baixo $(<16)$ & $33(30.3 \%)$ & $58(29.5 \%)$ & $115(34.0 \%)$ & \\
\hline Médio (17-19) & $28(25.7 \%)$ & $54(27.4 \%)$ & $94(27.8 \%)$ & \\
\hline Alto $(=20)$ & $47(43.1 \%)$ & $82(41.6 \%)$ & $125(37.0 \%)$ & \\
\hline Não responderam & $1(0.9 \%)$ & $3(1.5 \%)$ & $4(1.2 \%)$ & \\
\hline
\end{tabular}

Nota: AAVDs - Atividades avançadas de vida diária; AIVDs: Atividades instrumentais de vida diária. a Sign para $\mathrm{p}=0.021$ $\left(\chi^{2}=7.71 ; \mathrm{GL}=2\right) ;$ b Sign para $\mathrm{p}=0.015\left(\chi^{2}=8.42 ; \mathrm{GL}=2\right)$.

com maior estresse houve uma frequência mais alta de relatos de 3 ou mais doenças, fadiga e dependência parcial para em AIVD do que nos grupos com menor satisfação e menor estresse e não cuidadores. Independentemente do nível de estresse relatado, o grupo de cuidadores com menor satisfação superou os grupos de cuidadores e de não cuidadores quanto à frequência de sintomas de insônia.

Entre os cuidadores com menor estresse e os não cuidadores foram encontrados os maiores percentuais de idosos sem queixas de insônia. Entre os não cuidadores com menor satisfação foram registradas as frequências significantemente mais altas de idosos com 6 ou mais sintomas depressivos e com baixo nível de suporte social percebido.

Os idosos cuidadores e não cuidadores com maior satisfação na vida, cerca da metade, relataram ter 1 ou 2 doenças, ultrapassando significantemente os que relataram ter 3 ou mais. Da mesma forma, houve um percentual significativamente mais alto de não cuidadores e de cuidadores com maior satisfação e menor estresse que relataram não ter nenhuma doença em comparação com os que tinham maior estresse.

As menores frequências de idosos que pontuaram para fadiga, sintomas depressivos, insônia e dependência nas AIVD foram observadas 
Tabela 2. Idosos com menor e com maior satisfação, menos e mais estressados pelo cuidar e não-cuidadores, com maior e menor satisfação, conforme as condições de saúde, a funcionalidade em AIVD, o envolvimento em AAVD e o suporte social percebido. Estudo FIBRA em Campinas, Parnaíba e Ivoti, 2008-2009.

\begin{tabular}{|c|c|c|c|}
\hline \multirow[b]{3}{*}{ Variáveis } & \multicolumn{3}{|c|}{ Menor satisfação com a vida } \\
\hline & \multicolumn{2}{|c|}{ Cuidadores } & \multirow{2}{*}{$\begin{array}{c}\text { Não cuidadores } \\
(n=166)\end{array}$} \\
\hline & $\begin{array}{l}\text { Menor estresse } \\
\quad(n=59)\end{array}$ & $\begin{array}{l}\text { Maior estresse } \\
\quad(n=68)\end{array}$ & \\
\hline \multicolumn{4}{|l|}{ Número de Doenças } \\
\hline Zero & $2(3.3 \%)$ & $14(12.6 \%)$ & $10(06.0 \%)$ \\
\hline 1 ou 2 & $29(49.2 \%)$ & $39(35.1 \%)$ & $43(48.2 \%)$ \\
\hline 3 ou mais & $28(47.5 \%)$ & $58(52.3 \%)^{\mathrm{a}}$ & $30(45.8 \%)$ \\
\hline \multicolumn{4}{|l|}{ Fadiga } \\
\hline Não & $44(74.6 \%)$ & $69(62.2 \%)$ & $115(69.3 \%)$ \\
\hline Sim & $14(23.7 \%)$ & $41(36.9 \%)^{b}$ & $50(30.1 \%)$ \\
\hline Não responderam & $1(1.7 \%)$ & $1(0.9 \%)$ & $1(0.6 \%)$ \\
\hline \multicolumn{4}{|l|}{ Sintomas depressivos } \\
\hline Não $(\leq 5)$ & $45(76.3 \%)$ & $80(72.1 \%)$ & $114(68.7 \%)$ \\
\hline $\operatorname{Sim}(\geq 6)$ & $14(23.7 \%)$ & $31(27.9 \%)$ & $52(31.3 \%)^{\mathrm{d}}$ \\
\hline \multicolumn{4}{|l|}{ Insônia } \\
\hline Não & $19(32.2 \%)$ & $31(27.9 \%)$ & $68(41.0 \%)$ \\
\hline Sim & $40(67.8 \%)^{c}$ & $80(72.1 \%)^{c}$ & $98(59.0 \%)$ \\
\hline \multicolumn{4}{|l|}{ AAVD Interrompidas } \\
\hline$<2$ & $8(13.6 \%)$ & $22(19.8 \%)$ & $43(25.9 \%)$ \\
\hline $2-3$ & $33(55.9 \%)$ & $57(51.4 \%)$ & $68(41.0 \%)$ \\
\hline$>3$ & $18(30.5 \%)$ & $32(28.8 \%)$ & $55(33.1 \%)$ \\
\hline \multicolumn{4}{|l|}{ AIVD } \\
\hline Dep. Parcial & $25(42.4 \%)$ & $60(54.1 \%)^{e}$ & $63(38.0 \%)$ \\
\hline Independência & $34(57.6 \%)$ & $51(45.9 \%)$ & $102(61.4 \%)$ \\
\hline Não responderam & $0(0.0 \%)$ & $0(0.0 \%)$ & $1(0.6 \%)$ \\
\hline \multicolumn{4}{|l|}{ Suporte Social } \\
\hline Baixo $(\leq 16)$ & $21(35.6 \%)$ & $37(33.3 \%)$ & $73(44.5 \%)^{\mathrm{f}}$ \\
\hline Moderado (17-19) & $16(27.1 \%)$ & $27(24.3 \%)$ & $47(28.6 \%)$ \\
\hline Alto $(=20)$ & $21(35.6 \%)$ & $44(39.7 \%)$ & $44(26.8 \%)$ \\
\hline Não responderam & $1(1.7 \%)$ & $3(2.7 \%)$ & $2(1.2 \%)$ \\
\hline
\end{tabular}

nos grupos de cuidadores com maior satisfação combinada com menor estresse e no grupo de não cuidadores com maior satisfação. No grupo cuidadores com maior satisfação e menor estresse foi observada frequência superior de idosos com alto nível de suporte social percebido. Para o grupo com maior satisfação e maior estresse foi apontado qualidade intermediária do suporte social de que dispunham.

A Tabela 3 apresenta os dados das análises da regressão logística univariada que estimaram as probabilidades de associação independente entre baixa satisfação com a vida e gênero, idade, renda familiar, condições de saúde, intensidade do estresse vivenciado pelos cuidadores, funcionalidade, envolvimento em AAVD e suporte social percebido entre os cuidadores e os não cuidadores. Em nenhum dos grupos, foram observadas associações estatisticamente significantes entre baixa satisfação insônia, sintomas depressivos, 3 ou mais doenças crônicas, fadiga e baixo suporte social percebido. No grupo de não cuidadores, ter 1 ou 2 doenças crônicas também se associou com baixa satisfação. No entanto, as associações entre baixa satisfação e insônia, 3 ou mais doenças e sintomas depressivos foram mais robustas no grupo de não cuidadores. Alta intensidade do estresse associado ao cuidado não se associou com baixa satisfação entre os cuidadores, assim como as variáveis sociodemográficas em ambos os grupos.

A análise multivariada de regressão logística revelou associações entre baixa satisfação e insônia $(\mathrm{OR}=2,56), 6$ ou mais sintomas depressivos (OR $=2,18)$ e fadiga $(\mathrm{OR}=2,00)$ entre os cuidadores. Entre os não cuidadores foram observadas associações entre baixa satisfação e fadiga $(\mathrm{OR}=3,12), 6$ 


\begin{tabular}{|c|c|c|c|c|}
\hline \multirow[b]{3}{*}{ Variáveis } & \multicolumn{3}{|c|}{ Maior satisfação com a vida } & \multirow[b]{3}{*}{$\mathbf{p}$} \\
\hline & \multicolumn{2}{|c|}{ Cuidadores } & \multirow{2}{*}{$\begin{array}{c}\text { Não cuidadores } \\
(n=160)\end{array}$} & \\
\hline & $\begin{array}{l}\text { Menor estresse } \\
\quad(\mathrm{n}=49)\end{array}$ & $\begin{array}{l}\text { Maior estresse } \\
\quad(\mathbf{n}=\mathbf{8 3})\end{array}$ & & \\
\hline Número de Doenças & & & & 0.005 \\
\hline Zero & $8(16.3 \%)^{a}$ & $10(12.1 \%)$ & $26(16.3 \%)^{\mathrm{a}}$ & \\
\hline 1 ou 2 & $25(51.0 \%)^{a}$ & $43(51.8 \%)^{\mathrm{a}}$ & $81(50.6 \%)^{a}$ & \\
\hline 3 ou mais & $16(32.7 \%)$ & $30(36.1 \%)$ & $53(33.1 \%)$ & \\
\hline Fadiga & & & & $<0.001$ \\
\hline Não & $41(83.7 \%)^{b}$ & $67(80.7 \%)$ & $140(87.4 \%)^{b}$ & \\
\hline $\operatorname{Sim}$ & $7(14.3 \%)$ & $15(18.1 \%)$ & $17(10.7 \%)$ & \\
\hline Não responderam & $1(2.0 \%)$ & $1(1.2 \%)$ & $3(1.9 \%)$ & \\
\hline Sintomas depressivos & & & & $<0.001$ \\
\hline Não $(\leq 5)$ & $48(98.0 \%)^{d}$ & $70(84.3 \%)$ & $145(90.6 \%)^{d}$ & \\
\hline $\operatorname{Sim}(\geq 6)$ & $1(2.0 \%)$ & $13(15.6 \%)$ & $15(9.4 \%)$ & \\
\hline Insônia & & & & $<0.001$ \\
\hline Não & $31(63.3 \%)^{c}$ & $41(49.4 \%)$ & $91(56.9 \%)^{c}$ & \\
\hline Sim & $18(36.7 \%)$ & $42(50.6 \%)$ & $69(43.1 \%)$ & \\
\hline AAVD Interrompidas & & & & 0.270 \\
\hline$<2$ & $10(20.4 \%)$ & $20(24.1 \%)$ & $48(30.0 \%)$ & \\
\hline $2-3$ & $22(44.9 \%)$ & $36(43.4 \%)$ & $73(45.6 \%)$ & \\
\hline$>3$ & $17(34.7 \%)$ & $27(32.5 \%)$ & $39(24.4 \%)$ & \\
\hline AIVD & & & & 0.004 \\
\hline Dep. Parcial & $15(30.6 \%)$ & $32(38.6 \%)$ & $49(30.6 \%)$ & \\
\hline Independência & $34(69.4 \%)^{e}$ & $51(61.4 \%)$ & $111(69.4 \%)^{e}$ & \\
\hline Não responderam & $0(0.0 \%)$ & $0(0.0 \%)$ & $0(0.0 \%)$ & \\
\hline Suporte Social & & & & 0.001 \\
\hline Baixo $(\leq 16)$ & $12(24.5 \%)$ & $18(21.7 \%)$ & $37(23.2 \%)$ & \\
\hline Moderado (17-19) & $12(24.5 \%)$ & $27(32.5 \%)^{\mathrm{f}}$ & $47(29.5 \%)$ & \\
\hline Alto $(=20)$ & $25(51.0 \%)^{\mathrm{f}}$ & $38(45.8 \%)$ & $75(47.1 \%)$ & \\
\hline Não responderam & $0(0.0 \%)$ & $0(0.0 \%)$ & $1(0.6 \%)$ & \\
\hline
\end{tabular}

Nota: AAVDs - Atividades avançadas de vida diária; AIVDs: Atividades instrumentais de vida diária. ${ }^{a}$ Sign para $\mathrm{p}=0.005\left(\chi^{2}=\right.$ $25.22 ; \mathrm{GL}=10)$; ${ }^{\mathrm{b}} \operatorname{Sign}$ para $\mathrm{p}<0.001\left(\chi^{2}=33.50 ; \mathrm{GL}=5\right) ;{ }^{\mathrm{c}} \operatorname{Sign}$ para $\mathrm{p}<0.001\left(\chi^{2}=34.46 ; \mathrm{GL}=5\right) ;{ }^{\mathrm{d}}$ Sign para $\mathrm{p}<0.001\left(\chi^{2}=\right.$ $40.23 ; \mathrm{GL}=5) ;{ }^{\mathrm{e}}$ Sign para $\mathrm{p}=0.004\left(\chi^{2} 2=17.09 ; \mathrm{GL}=5\right) ;{ }^{\mathrm{f}} \operatorname{Sign}$ para $\mathrm{p}=0.001\left(\chi^{2}=28.90 ; \mathrm{GL}=10\right)$.

ou mais sintomas depressivos $(\mathrm{OR}=2,85)$ e baixo suporte social percebido $(\mathrm{OR}=1,78)$, todas mais fortes do que entre os cuidadores (Tabela 4).

\section{Discussão}

Confirmando os resultados de outros estudos sobre bem-estar subjetivo na velhice, a maioria dos idosos mostrou-se satisfeito com a vida ${ }^{27}$, independentemente de serem cuidadores $^{10}$, assim como confirmaram-se os resultados de pesquisas sobre os mecanismos de adaptação psicológica presentes na velhice ${ }^{1}$. De acordo com a microteoria de seletividade socioemocional ${ }^{7,8}$, em função da redução da perspectiva de tempo futuro que ocorre na velhice, entre os idosos diminui o interesse por experiências emocionais negativas, aumenta o investimento em metas de maior significado afetivo e ocorre seleção de parceiros sociais que oferecem mais conforto emocional. Tais processos reguladores da emoção favorecem o equilíbrio entre afetos positivos e negativos, a estabilidade emocional e a manutenção de níveis elevados de bem-estar subjetivo, que os protegem da ação deletéria dos eventos estressores ${ }^{28}$.

À semelhança da maioria dos estudos sobre o cuidado, observou-se que entre os cuidadores predominavam mulheres, que dispensavam apoio principalmente aos maridos ${ }^{29}$, de idosos de 65 e 79 anos, e de idosos de baixa renda ${ }^{30}$. O prestar cuidados é regido por normas culturais que atribuem às mulheres as tarefas e os papeis de proteção. Em muitos contextos, as mulheres são mais jovens e mais pobres do que os maridos, o que fortalece a probabilidade de que venham a cuidar deles. Os 
Tabela 3. Associações independentes entre baixa satisfação com a vida e as variáveis sociodemográficas, de saúde e funcionalidade, envolvimento social e suporte social para cuidadores e não cuidadores e entre baixa satisfação com a vida e intensidade do estresse associado ao cuidado para cuidadores. Estudo FIBRA em Campinas, Parnaíba e Ivoti, idosos, 2008-2009.

\begin{tabular}{|c|c|c|c|c|}
\hline \multirow[b]{2}{*}{ Categorias } & \multicolumn{2}{|c|}{ Cuidadores } & \multicolumn{2}{|c|}{ Não cuidadores } \\
\hline & $\mathbf{p}$ & $\mathrm{OR}^{*}(\mathrm{IC} 95 \%)$ & $\mathbf{p}$ & $\mathrm{OR}^{*}(\mathrm{IC}$ 95\%) \\
\hline \multicolumn{5}{|l|}{ Gênero } \\
\hline Feminino & 0.943 & $1.02(0.64-1.61)$ & 0.918 & $0.98(0.62-1.54)$ \\
\hline \multicolumn{5}{|l|}{ Faixa Etária } \\
\hline 65-69 anos (ref.) & --- & 1.00 & --- & 1.00 \\
\hline 70-74 anos & 0.275 & $0.76(0.46-1.25)$ & 0.405 & $1.23(0.75-2.03)$ \\
\hline 75-79 anos & 0.556 & $0.83(0.44-1.56)$ & 0.742 & $1.11(0.59-2.12)$ \\
\hline$\geq 80$ anos & 0.883 & $1.07(0.43-2.65)$ & 0.375 & $0.66(0.27-1.64)$ \\
\hline \multicolumn{5}{|l|}{ Renda Familiar } \\
\hline > 5.0 S.M. (ref.) & --- & 1.00 & --- & 1.00 \\
\hline $.1-5.0$ S.M. & 0.964 & $1.02(0.51-2.04)$ & 0.741 & $1.59(0.67-3.79)$ \\
\hline $.1-3.0$ S.M. & 0.793 & $1.08(0.59-1.98)$ & 0.145 & $2.23(0.86-5.77)$ \\
\hline 1.0 S.M. & 0.146 & $2.12(0.77-5.87)$ & 0.061 & $2.52(0.96-6.62)$ \\
\hline \multicolumn{5}{|l|}{ Número de doenças } \\
\hline 0 (ref.) & --- & 1.00 & --- & 1.00 \\
\hline $1-2$ & 0.329 & $1.43(0.70-2.94)$ & 0.020 & $2.57(1.16-5.67)$ \\
\hline$\geq 3$ & 0.012 & $2.56(1.23-5.31)$ & 0.001 & $3.73(1.66-8.38)$ \\
\hline Presença de Fadiga & 0.004 & $2.21(1.30-3.76)$ & $<0.001$ & $3.58(1.96-6.54)$ \\
\hline Presença de Sintomas depressivos & $<0.001$ & $2.99(1.62-5.51)$ & $<0.001$ & $4.41(2.36-8.24)$ \\
\hline Presença Insônia & $<0.001$ & $3.21(2.03-5.07)$ & 0.004 & $1.90(1.22-2.95)$ \\
\hline \multicolumn{5}{|l|}{ AAVD Interrompidas } \\
\hline$<2$ (ref.) & --- & 1.00 & --- & 1.00 \\
\hline $2-3$ & 0.185 & $1.47(0.83-2.60)$ & 0.885 & $1.04(0.61-1.76)$ \\
\hline$>3$ & 0.570 & $1.20(0.65-2.21)$ & 0.126 & $1.57(0.88-2.82)$ \\
\hline Dependência em AIVD & 0.014 & $1.75(1.12-2.73)$ & 0.152 & $1.40(0.88-2.22)$ \\
\hline \multicolumn{5}{|l|}{ Suporte social percebido } \\
\hline$\geq 20$ (ref.) & --- & 1.00 & --- & 1.00 \\
\hline$\leq 17-19$ & 0.540 & $1.18(0.69-2.03)$ & 0.057 & $1.71(0.98-2.95)$ \\
\hline$\leq 16$ & 0.015 & $1.92(1.13-3.24)$ & $<0.001$ & $3.36(1.95-5.79)$ \\
\hline Intensidade do estresse do cuidar & 0.426 & $1.071(0.91-1.27)$ & & - \\
\hline
\end{tabular}

Nota: AAVDs - Atividades avançadas de vida diária; AIVDs: Atividades instrumentais de vida diária, SM - Salário Mínimo. * OR (Odds Ratio) = Razão de risco para satisfação com a vida; (cuidadores: $\mathrm{n}=186$ com menor satisfação e $\mathrm{n}=146$ com maior satisfação); (não cuidadores : $\mathrm{n}=166$ com menor satisfação e $\mathrm{n}=160 \mathrm{com}$ maior satisfação). IC 95\% OR $=$ Intervalo de $95 \%$ de confiança para a razão de risco. Ref.: nível de referência.

idosos jovens têm maior probabilidade de terem pais vivos e com possibilidade de precisarem de cuidados do que os idosos mais velhos. Da mesma forma, os mais pobres estão mais expostos à prestação de cuidado familiar, porque têm menos acesso a ajudas formais para cuidar.

A literatura não é consensual quanto às diferenças em condições de saúde, bem-estar subjetivo e recursos sociais entre cuidadores e não cuidadores. Algumas pesquisas não encontraram diferenças significativas entre os grupos, com relação a sintomas depressivos e à participação social (por exemplo, Robison et al. ${ }^{31}$ ) e observaram nível similar de satisfação com a vida entre cuidadores menos comprometidos com o cuidado e não cuidadores (por exemplo, Borg e Hallberg ${ }^{10}$ ), contrariando outras que apresentam diferenças em indicadores de saúde e bem estar subjetivo, como número de doenças ${ }^{32}$, sintomas depressivos, satisfação com a vida ${ }^{32,33}$ e suporte social ${ }^{10}$, com prejuízo para os cuidadores. Há dados indicativos de que altos escores de suporte social percebido e de satisfação com a vida tendem a funcionar como variáveis protetoras em relação ao bem estar dos cuidadores ${ }^{1,5,10,34}$.

A exploração das relações entre satisfação com a vida, prestação de cuidado e nível de estresse em idosos foi pouco estudada na literatura nacional. 
Tabela 4. Associações múltiplas entre baixa satisfação, insônia, sintomas depressivo, fadiga e suporte social percebido para idosos cuidadores e não cuidadores. Estudo Fibra em Campinas, Parnaíba e Ivoti, 2008-2009.

\begin{tabular}{|c|c|c|c|c|}
\hline \multirow[b]{2}{*}{ Variáveis selecionadas } & \multicolumn{2}{|c|}{ Cuidadores } & \multicolumn{2}{|c|}{ Não cuidadores } \\
\hline & $\mathbf{p}$ & $\mathrm{OR}^{*}(\mathrm{IC}$ 95\%) & $\mathbf{p}$ & $\mathrm{OR}^{*}(\mathrm{IC}$ 95\%) \\
\hline \multicolumn{5}{|l|}{ Insônia } \\
\hline Não (ref) & --- & 1,00 & --- & --- \\
\hline Sim & $<0.001$ & $2.56(1.51-4.32)$ & & \\
\hline \multicolumn{5}{|l|}{ Sintomas depressivos } \\
\hline Não (ref) & --- & 1,00 & --- & 1,00 \\
\hline Sim & 0.032 & $2,18(1,07-4,43)$ & 0.002 & $2.85(1.46-5.57)$ \\
\hline \multicolumn{5}{|l|}{ Fadiga } \\
\hline Não (ref) & --- & 1,00 & 1,00 & 1,00 \\
\hline Sim & 0.036 & $2.00(1.05-3.82)$ & $<0.001$ & $3.12(1.62-6.02)$ \\
\hline \multicolumn{5}{|l|}{ Suporte social percebido } \\
\hline$\geq 20$ (ref.) & --- & --- & --- & 1.00 \\
\hline $17-19$ & & & 0.340 & $1.35(0.73-2.50)$ \\
\hline$\leq 16$ & & & 0.004 & $2.43(1.32-4.47)$ \\
\hline
\end{tabular}

* OR (Odds Ratio) = Razão de risco para satisfação com a vida; (cuidadores: $\mathrm{n}=149$ com menor satisfação e $\mathrm{n}=118$ com maior satisfação); (não cuidadores: $\mathrm{n}=147$ com menor satisfação e $\mathrm{n}=140$ com maior satisfação). IC 95\% OR $=$ Intervalo de $95 \%$ de confiança para a razão de risco. Critério stepwise de seleção de variáveis.

Nesta pesquisa, escores mais baixos de satisfação combinados com alto nível de estresse relativo ao cuidado relacionaram-se com ter 3 ou mais doenças, fadiga, insônia e dependência parcial para mais AIVD. Além disso, insônia esteve relacionada com o prestar cuidados, independentemente do nível de estresse, replicando dados de Borg e Hallberg $^{10}$. A ligação entre estresse e nível mais baixo de satisfação com a vida tem sido constantemente encontrada em vários estudos ${ }^{35-37}$. É provável que a concomitância de menor satisfação com a vida e maior estresse contribuam para pior condição de saúde, mais incapacidades em AIVD e maior fadiga entre cuidadores.

Não foram observadas diferenças estatisticamente significantes entre a frequência de idosos de nenhum dos grupos, quanto ao nível de estresse e nem conforme a satisfação com a vida, levandose em conta o número de AAVD descontinuadas. Havia mais idosos não cuidadores com menor satisfação e baixo suporte social e mais cuidadores com menos estresse com alto suporte social, assim como havia maior número de cuidadores com maior satisfação e maior estresse entre os que pontuaram para suporte social percebido de qualidade intermediária. Talvez esses dados se devam à influência da idade e não da privação de contatos sociais por causa do prestar cuidados. Geralmente os indivíduos mais velhos participam menos de eventos sociais do que os mais jovens, por encontrarem mais barreiras ambientais ou por se sentirem menos ativos e habilitados para a realização de atividades sociais ${ }^{38}$. Idosos tendem a afastar-se de atividades menos significativas, a favor de outras que lhes tragam conforto emocional ${ }^{39}$.

A análise multivariada de regressão mostrou que as variáveis mais fortemente associadas com menor satisfação com a vida entre os cuidadores foram sintomas de insônia, sintomas depressivos, ter 3 ou mais doenças crônicas e fadiga, concordando com outros estudos ${ }^{10,40,41}$. Provavelmente, essas relações possam ser explicadas pelas pressões associadas ao cuidado ${ }^{42}$. Entretanto, as alterações do sono, os sintomas depressivos e a fadiga são inter-relacionados, sendo difícil identificar os determinantes e os efeitos separados de cada uma delas $^{41}$. Sintomas depressivos, fadiga e 3 ou mais doenças não só se associaram à baixa satisfação entre os não cuidadores, como as associações foram mais fortes neste grupo do que dos cuidadores. Ao mesmo tempo, contrariando as expectativas, baixo suporte social percebido associou-se à baixa satisfação no grupo de não cuidadores, e não no grupo de cuidadores.

As análises logísticas univariada e multivariada não apontaram a intensidade do estresse relacionado ao cuidado como condição associada à menor satisfação com a vida entre os cuidadores, replicando dados de Lee et al. ${ }^{36}$, mas diferindo do estudo realizado por Andrén e Elmståhl ${ }^{12}$. Talvez essa relação sofra diversas influências, entre elas a capacidade de enfrentamento e a idade, que pode se sobrepor aos efeitos do prestar cuidados. Segundo Ng et al. ${ }^{37}$ quando a intensidade dos estressores 
é compatível com as reservas afetivas e cognitivas dos envolvidos, o sucesso no enfrentamento de tarefas desafiadoras e, em consequência, a estabilidade da satisfação, tornam-se mais prováveis, o que explicaria a frequência alta de idosos que pontuou para alta satisfação no grupo de cuidadores, igualando a dos não cuidadores. Mecanismos de seletividade sócio-emocional que contribuem para a estabilidade emocional, permitem que os idosos poupem suas reservas para investimento em alvos sociais, emocionais e cognitivos relevantes à sua adaptação à vida prática ${ }^{7,8}$.

As associações entre baixa satisfação e as variáveis idade, gênero e renda familiar não foram suficientemente robustas para suplantar ou igualar o peso das variáveis de saúde em nenhum dos grupos. Enkvist et al. ${ }^{6}$ observaram que a idade avançada é um fator de risco para baixa satisfação com a vida, enquanto que Gana et al. ${ }^{14}$ e Sposito et al. ${ }^{43}$ não observaram tal associação. Os dados desta pesquisa foram similares aos de Enkvist et al. ${ }^{6} \mathrm{e}$ Gana et al. ${ }^{14}$, no que diz respeito ao gênero. É provável que o envolvimento social sofra influência da idade, uma vez que, geralmente os indivíduos mais velhos participam menos de eventos sociais do que os mais jovens por encontrarem mais barreiras ambientais e por se sentirem menos ativos e habilitados para a realização de atividades sociais ${ }^{36}$.

\section{Conclusões}

Entre os cuidadores, sintomas de insônia, sintomas depressivos, 3 ou mais doenças e fadiga associaram-se simultaneamente à baixa satisfação com a vida, suplantando a influência isolada das variáveis baixo suporte social percebido e dependência parcial em AIVD, provavelmente pelo fato de os cuidadores serem majoritariamente mulheres, mais doentes e com mais limitações funcionais por causa de sua condição feminina. As associações múltiplas mais robustas observadas entre não cuidadores do que entre cuidadores, envolvendo baixa satisfação, fadiga e sintomas depressivos e baixa satisfação e o fato de o suporte social ter se associado com baixa satisfação apenas entre os cuidadores, sugerem que essas variáveis são mais relevantes ao bem-estar subjetivo deste grupo do que daquele. Esta interpretação exige cautela expressa em mais estudos, pois outras variáveis podem estar mediando essas relações.

Os dados e as interpretações derivadas da presente investigação são limitadas pelo desconhecimento das circunstâncias do cuidado, incluindo a duração do cuidado, suas demandas especificas e o grau de envolvimento dos idosos com as tarefas de cuidar. Estas são influenciadas pelo status de saúde; pelo estágio e o prognóstico da doença do ente cuidado; pela qualidade das relações familiares; pelas estratégias de enfrentamento de eventos estressores adotadas pelos cuidadores; pelo nível de ajustamento do self e pelos traços de personalidade dessas pessoas, e pela quantidade e a qualidade do apoio formal recebido, entre outros fatores. Além disso, o estudo envolveu apenas variáveis de autorrelato, exposto à influência de vieses de memória e de avaliação e não contou com o acompanhamento dos participantes ao longo do tempo.

A despeito das limitações, cabe ressaltar sua importância como um dos poucos estudos brasileiros relativos à descrição de relações entre saúde, funcionalidade, envolvimento social, suporte social e bem-estar de cuidadores familiares, principalmente considerando que seus participantes são idosos cuidando de outros idosos, uma condição em crescimento na população brasileira, com todas as implicações financeiras, sociais e éticas implicadas nessa relação. Novas pesquisas poderão ser realizadas com controles das condições apontadas e com acompanhamento longitudinal. Profissionais brasileiros que planejam e implementam programas de intervenção psicológica, educacional, social e de saúde, dirigidos a idosos cuidadores e à sua rede de suporte, poderão beneficiar-se dos dados aqui apresentados, que são sugestivos de novas e produtivas formas de observar e analisar os fenômenos envolvidos. 


\section{Colaboradores}

MRSV Tomomitsu e MR Perracini foram responsáveis pelo desenho do estudo, análise dos dados e redação do artigo. AL Neri é a coordenadora do principal projeto de pesquisa e colaborou na concepção do estudo, na análise de dados e na revisão final do manuscrito.

\section{Agradecimentos}

Agradecemos aos colaboradores L.F. Araújo, G.A. Santos, A.C.G. Fortes-Burgos, E.P. Mantovani, F.S. Arbex Borim e S.V.S. Torres pela coordenação dos processos de recrutamento e coleta de dados em Parnaíba, Ivoti e Campinas.

\section{Referências}

1. Diener E, Ryan K. Subjective well-being: a general overview. S Afr J Psychol 2009; 39(4):391-406.

2. Fujita F, Diener E. Life satisfaction set point: stability and change. J Pers Soc Psychol 2005; 88(1):158-164.

3. Mroczek DK, Spiro A. Change in life satisfaction over 20 years during adulthood: findings from the VA Normative Aging Study. J Personal Soc Psychol 2005; 88(1):189-202.

4. Gerstorf D, Ram N, Mayraz G, Hidajat M, Lindenberger U, Wagner GG, Schupp J. Late-life decline in well-being across adulthood in Germany, the United Kingdom, and the United States: Something is seriously wrong at the end of life. Psychol Aging 2010; 25(2):477-485.

5. Hernandez CR, López V, Ortiz T. Satisfaction with life related to functionality in active elderly people. Actas Esp Psiquiatr 2009; 37(2):61-67.

6. Enkvist A, Ekström H, Elmståhl S. What factors affect life satisfaction (LS) among the oldest-old? Arch Gerontol Geriatr 2012; 54(1):140-145.

7. Carstensen LL, Fung HH, Charles ST. Socioemotional selectivity theory and the regulation of emotion in the second half of life. Motivation and emotion 2003; 27(2):103-123.

8. Carstensen LL, Turan B, Scheibe S, Ram N, Ersner-Hershfield H, Samanez-Larkin GR, Brooks KP, Nesselroade JR. Emotional Experience Improves With Age: Evidence Based on Over 10 Years of Experience Sampling. Psychol Aging 2011; 26(1): 21-33.

9. Pinquart $M$, Sörensen $S$. Associations of stressors and uplifts of caregiving with caregiver burden and depressive mood: a meta-analysis. J Gerontol B Psychol Sci Soc Sci 2004; 58(2):112-128

10. Borg C, Hallberg IR. Life satisfaction among informal caregivers in comparison with non-caregivers. Scand J Caring Sci 2006; 20(4):427-438.

11. Haley WE, LaMonde LA, Han B, Burton AM, Schonwetter R. Predictors of depression and life satisfaction among spousal caregivers in hospice: application of a stress process model. J Palliat Med 2003; 6(2):215-224.

12. Andrén $S$, Elmståhl $S$. The relationship between caregiver burden, caregivers' perceived health and their sense of coherence in caring for elders with dementia. J Clin. Nurs 2008; 17(6):790-799.

13. Enkvist Å, Ekström H, Elmståhl S. Associations between functional ability and life satisfaction in the oldest old: results from the longitudinal population study Good Aging in Skåne. Clin Interv Aging 2012; 7:313-320.

14. Gana K, Bailly N, Saada Y, Joulain M, Alaphilippe D. Does Life Satisfaction Change in Old Age: Results From an 8-Year Longitudinal Study. J Gerontol B Psychol Sci Soc Sci 2013; 68(4):540-552

15. Brucki SM, Nitrini R, Caramelli P, Bertolucci PH, Okamoto IH. Suggestions for utilization of the mini-mental state examination in Brazil. Arq Neuropsiquiatr 2003; 61(3):777-781.

16. Neri AL, Yassuda MS, de Araújo LF, Eulálio Mdo C, Cabral BE, de Siqueira ME, dos Santos GA, Moura JG. Metodologia e perfil sociodemográfica cognitive e de fragilidade de idosos comunitários de sete cidades brasileiras: Estudo FIBRA. Cad Saude Publica 2013; 29(4):778-792.

17. Fortes-Burgos ACG, Neri AL, Cupertino A. Eventos estressantes, estratégias de enfrentamento, auto-eficácia e sintomas depressivos entre idosos residentes na comunidade. Psicologia: Reflexão e Crítica 2008; 21(1):74-82. 
18. Fortes-Burgos ACG, Neri AL. Experiência de eventos estressantes. In: Neri AL, Guariento ME, organizadores. Fragilidade, saúde e bem-estar em idosos: Dados do estudo FIBRA Campinas. Campinas: Alínea; 2011. p. 225-238.

19. Neri AL. Bienestar subjetivo en la vida adulta y en la vejez: rumbo a una Psicologia positiva en América Latina. Revista Latinoamericana de Psicologia 2002; 43(1-2):55-74.

20. Batistoni S, Neri A, Cupertino A. Validade da escala de depressão do Center for Epidemiological Studies entre idosos brasileiros. Rev Saude Publica 2007; 41(4):598605.

21. Almeida O, Almeida S. Short versions of the geriatric depression scale: a study of their validity for the diagnosis of a major depressive episode according to ICD-10 and DSM-IV. Int J Geriatr Psychiatry 1999; 14(10):858-865.

22. Teixeira-Salmela LF, Magalhães LC, Souza AC, Lima MC, Lima RC, Goulart F. Adaptation of the Nottingham Health Profile: A simple measure to assess quality of life. Cad Saude Publica 2004; 20(4):905-914.

23. Lawton M, Brody E. Assessment of older people: self-maintaining and instrumental activities of daily living. Gerontologist 1969; 9(3):179-186.

24. Strawbridge W, Wallhagen M, Cohen R. Successful aging and well-being: self-rated compared with Rowe and Kahn. Gerontologist 2002; 42(6):727-733.

25. Reuben DB, Laliberte L, Hiris J, Mor V. A hierarchical exercise scale to measure function at the Advanced Activities of Daily Living (AADL) level. J Am Geriatr Soc 1990; 38(8):855-861.

26. Martire L, Schulz R, Mittelmark M, Newsom J. Stability and change in older adults' social contact and social support: the Cardiovascular Health Study. J Gerontol B Psychol Sci Soc Sci 1999; 54(5):S302-S311.

27. Strine TW, Chapman DP, Balluz LS, Moriarty DG, Mokdad AH. The associations between life satisfaction and health-related quality of life, chronic illness, and health behaviors among U.S. community-dwelling adults. $J$ Community Health 2008; 33(1):40-50.

28. Scheibe S, Carstensen LL. Emotional aging: recent findings and future trends. J Gerontol B Psychol Sci Soc Sci 2010; 65B(2):135-144.

29. Roberto KA, Jarrot SE. Family Caregivers of Older Adults: A Life Span Perspective. Family Relations 2008; 57(1):100-111.

30. Pinquart M, Sörensen S. Spouses, adult children, and children-in-law as caregivers of older adults: a meta-analytic comparison. Psychol Aging 2011; 26(1):1-14.

31. Robison J, Fortinsky R, Kleppinger A, Shugrue N, Porter $\mathrm{M}$. A broader view of family caregiving: effects of caregiving and caregiver conditions on depressive symptoms, health, work, and social isolation. J Gerontol B Psychol Sci Soc Sci 2009; 64(6):788-798.
32. Pinquart M, Sörensen S. Differences between caregivers and noncaregivers in psychological health and physical health: a meta-analysis. Psychol Aging 2003; 18(2):250-267.

33. Ostwald SK, Godwin KM, Cron SG. Predictors of life satisfaction in stroke survivors and spousal caregivers after inpatient rehabilitation. Rehabil Nurs 2009; 34(4):160-167.

34. Hsu HC. Physical function trajectories, depressive symptoms, and life satisfaction among the elderly in Taiwan. Aging Ment Health 2009; 13(2):202-212.

35. Pinquart M, Sörensen S. Predictors of caregiver burden and depressive mood: a meta-analysis. J Gerontol Psychol Sci 2003; 58(2):112-128.

36. Lee CC, Czaja SJ, Schulz R. The moderating influence of demographic characteristics, social support, and religious coping on the effectiveness of a multicomponent psychosocial caregiver intervention in three racial ethnic groups. J Gerontol B Psychol Sci Soc Sci 2010; 65B (2):185-194

37. Ng W, Diener E, Aurora R, Harter J. Affluence, Feelings of stress, and well-being. Soc Indic Res 2009; 94(2):257-271.

38. Levasseur M, Desrosiers J, Whiteneck G. Accomplishment level and satisfaction with social participation of older adults: association with quality of life and best correlates. Qual Life Res 2010; 19(5):665-675.

39. Pinto JM, Neri AL. Doenças crônicas, capacidade funcional, envolvimento social e satisfação em idosos comunitários: Estudo Fibra. Cien Saude Colet 2013; 18(12):3449-3460.

40. Staner L. Comorbidity of insomnia and depression. Sleep Med Rev 2010; 14(1):35-46.

41. Goldman SE, Ancoli-Israel S, Boudreau R, Cauley JA, Hall M, Stone KL, Rubin SM, Satterfield S, Simonsick EM, Newman AB; Health, Aging and Body Composition Study. Sleep problems and associated daytime fatigue in community-dwelling older individuals. J Gerontol A Biol Sci Med Sci 2008; 63(10):1069-1075.

42. Goldsworthy B, Knowles S. Caregiving for Parkinson's disease patients: an exploration of a stress-appraisal model for quality of life and burden.J Gerontol B Psychol Sci Soc Sci 2008; 63(6):372-376.

43. Sposito G, D'elboux MJ, Neri AL, Guariento ME. A satisfação com a vida e a funcionalidade em idosos atendidos em um ambulatório de geriatria. Cien Saude Colet 2013; 18(12):3475-3482.

Artigo apresentado em 10/08/2013

Aprovado em 20/03/2014

Versão final apresentada em 23/03/2014 